\title{
IMPACT OF FREE MANEUVERING AIRCRAFT ON GROUND-SIDE OPERATIONS IN MIXED AIRSPACE
}

\author{
Paul U. Lee, Thomas Prevot, Joey Mercer, SJSU / NASA Ames Research Center, Moffett Field, CA \\ Nancy Smith, Everett A. Palmer, NASA Ames Research Center, Moffett Field, CA
}

\begin{abstract}
Numerous concepts aimed at increasing airspace capacity have been proposed to meet the anticipated increase in future air traffic demand. NASA Ames and NASA Langley Research Centers have recently conducted a joint simulation to test the En Route Free Maneuvering concept element of Distributed Air-Ground Traffic Management (DAG-TM), which integrated advanced air and ground decision support tools (DSTs) with Controller-Pilot Data Link Communication (CPDLC). In this concept, controller-"managed" aircraft flying under Instrument Flight Rules (IFR) were mixed with free maneuvering aircraft flying under Autonomous Flight Rules (AFR). Under AFR, free maneuvering aircraft were responsible for conflicts with all managed aircraft flying in the same airspace. The results showed a significant potential for capacity gains. The workload correlated primarily with the managed portion of the traffic in mixed operations and the analyses of the controller task load in mixed operations showed a significant reduction of "routine" tasks, such as check-ins and handoffs, as well as route, altitude, and speed clearances. Despite the reduction in workload in mixed operations, controllers had a number of safety concerns, such as over-reliance on automation and lack of situation awareness of AFR aircraft. The findings from the study also suggest that integrated ground-side DSTs in themselves have potential to increase capacity without free maneuvering aircraft. DAG-TM research was funded by the Airspace Systems program as part of the Advanced Air Transportation Technologies project. DAG-TM activities were conducted by NASA Ames, NASA Langley, and NASA Glen Research Center.
\end{abstract}

\section{Introduction}

The objective of Distributed Air/Ground Traffic Management (DAG-TM) was to investigate new concept of operations to meet the future demands of air travel [1]. One concept element within DAG-TM is En Route Free Maneuvering, which delegates the separation responsibilities to the flight crews of properly equipped aircraft. By distributing both the tasks and the responsibilities from controllers to flight crews, the concept aims at gaining significant en route capacity and improving efficiency. By eliminating the controller workload as a limiting factor to total aircraft capacity, the upper limit of capacity may be much higher, perhaps up to the physical airspace capacity limit. By allowing the flight crews to fly preferred routes and altitudes, they may fly routes optimal for fuel efficiency.

During the DAG-TM research, newly defined Autonomous Flight Rules (AFR) allowed the pilots of free maneuvering aircraft to choose their own routes, speeds, and altitudes without the controller's approval, as long as they do not create short-term conflicts and assume responsibility for separation from other self separating and managed traffic. The controllers were still responsible for separation between managed aircraft complying with standard Instrument Flight Rules (IFR) operations. En Route Free Maneuvering - like many other future air traffic concepts proposed by NASA, RTCA, Eurocontrol, etc. - proposes to distribute tasks and responsibilities using well integrated air-ground decision support tools (DSTs) [1-4]

In 2004, a joint human-in-the-loop experiment was conducted at NASA Ames and NASA Langley Research Center to investigate the feasibility and operational benefits of this concept [5]. The experiment addressed two primary issues: the feasibility of conducting mixed operations with autonomous and managed aircraft in the same airspace and the ability to scale the en route traffic capacity by increasing the autonomous portion of the air traffic without adversely effecting controller workload. The details of the simulation study are described in the following sections. 


\section{Method}

\section{Participants}

Subject participants consisted of 22 commercial airline pilots and 5 certified professional air traffic controllers. Four controllers staffed the radar positions (three high altitude sectors and one low altitude sector) and an additional controller served as a tracker supporting the radar controllers. Twenty one aircraft simulators were flown by participant pilots at NASA Ames and NASA Langley. All remaining aircraft in the simulation were flown by pseudo-pilots with autonomous agent support at Ames and Langley.

\section{Airspace}

The simulation airspace included modified portions of Albuquerque Center (ZAB), Fort Worth Center (ZFW) and Dallas-Fort Worth TRACON (DFW) (Figure 1). Controller participants worked three high altitude sectors (Amarillo in ZAB, Wichita Falls and Ardmore in ZFW), and one ZFW low altitude sector (Bowie). Three retired controllers worked Ghost positions to handle the surrounding traffic.

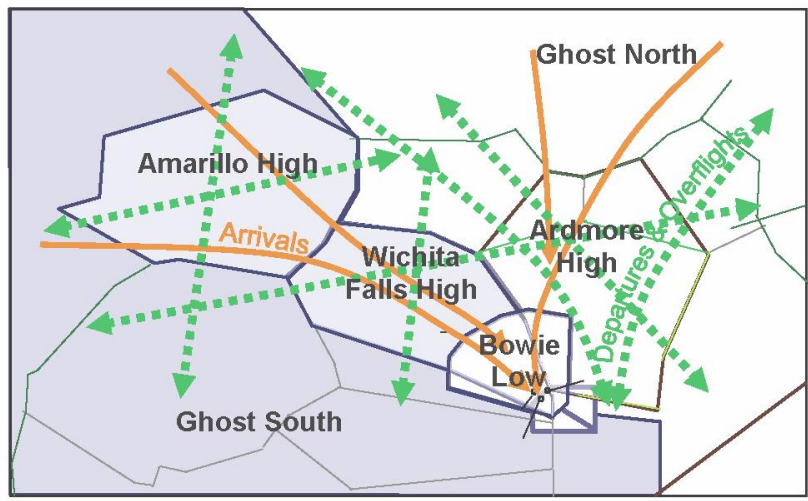

Figure 1. Simulated Airspace

The two main streams of arrivals merged at the BAMBE meter fix in the Bowie low sector before entering the TRACON. Once AFR aircraft passed the meter fix and were under TRACON control, their status switched to IFR automatically. The traffic mix in Amarillo consisted of en route aircraft in level flight. Both Wichita Falls and Ardmore had a mixture of arrivals, overflights, and departures, but Wichita Falls had mostly arrivals while Ardmore had more even distribution of flights.

\section{Experiment Design}

The experiment consisted of four conditions (Figure 2). Each condition was run five times, four of which were used in subsequent analyses.

Conditions $\mathrm{C} 1$ and $\mathrm{C} 2$ were conducted at above current day maximum traffic levels (Level 1 or L1), the former consisting of entirely managed aircraft and the latter having a mix of autonomous $(\sim 25 \%)$ and managed $(\sim 75 \%)$ aircraft

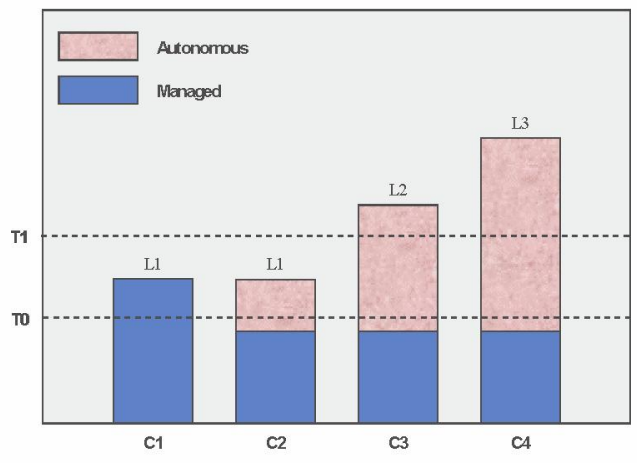

Figure 2. Experimental Design

Level 1 traffic levels were established for the three high altitude sectors through an informal study [6]. This informal "traffic load test" determined the maximum traffic levels for each of the high altitude sectors. The maximum manageable traffic levels came out higher than the current day Monitor Alert Parameters (MAPs), even with only one controller per sector, because the advanced DSTs alone offloaded the controller workload significantly. Level 1 traffic levels were then picked to be slightly lower than the maximum traffic count, resulting in traffic levels similar to current day MAPs (18 in each high altitude sector).

Conditions $\mathrm{C} 3$ and $\mathrm{C} 4$ included the same number of managed aircraft as Condition $\mathrm{C} 2$, but added increasing numbers of AFR aircraft. Traffic volumes were varied by altering the number of overflights. The traffic volume increase was greater for Amarillo and Ardmore than for Wichita Falls because the sector geometry of Wichita Falls prevented a significant increase in total aircraft count without significantly increasing the traffic complexity. The arrival problem, while demanding, remained relatively constant throughout all 
scenarios. Accordingly, Bowie sector, which had arrival traffic only, maintained a constant traffic volume across conditions.

The experiment tested the feasibility and benefits of both mixed operations and scalability. Operational viability of mixed operations was assessed by examining the impact of mixed operations on safety, efficiency, and controller workload. Scalability was assessed by examining safety, efficiency, and workload when the total number of aircraft in a sector is increased far beyond the number that a controller can safely managed if they were all controller-managed. The ground-side metrics - such as controller workload, acceptability ratings on operational concept/procedures, ground-side decision support tool ratings, controller safety ratings/concerns, meter fix conformance, number of operational errors, etc. - were used to assess the impact of the operational concept on the ground-side operations.

\section{Separation Responsibilities}

To achieve scalability, free maneuvering aircraft needed to have little or no impact on controller workload. A key concept designed to achieve this goal was that the pilot flying under AFR was responsible for separating their aircraft from all other aircraft, including controllermanaged IFR aircraft. The controller was only responsible for separation assurance between two or more IFR aircraft. To minimize the interactions between AFR and IFR aircraft, pilots of AFR aircraft were expected to resolve all conflicts at least 2 minutes before loss of separation (LOS) and the conflicts were shown to the controllers only if they were not resolved by 3 minutes before LOS. Controllers could contact the pilot to coordinate a resolution, ask for pilot's intent, etc., but they were not required to do so. In addition, pilots and controllers could not make flight path changes that caused a predicted LOS of less than 4 minutes. The minimum separation distance was 5 NM laterally and $1,000 \mathrm{ft}$ vertically (reduced vertical separation minimum).

\section{Ground Capabilities}

The controller decision support tools have been integrated into a high fidelity emulation of the
Display System Replacement (DSR) controller workstation (Figure 3). This DSR emulator is highly configurable to mimic both DSR workstations in the field today and future DSRs with advanced decision support tools.

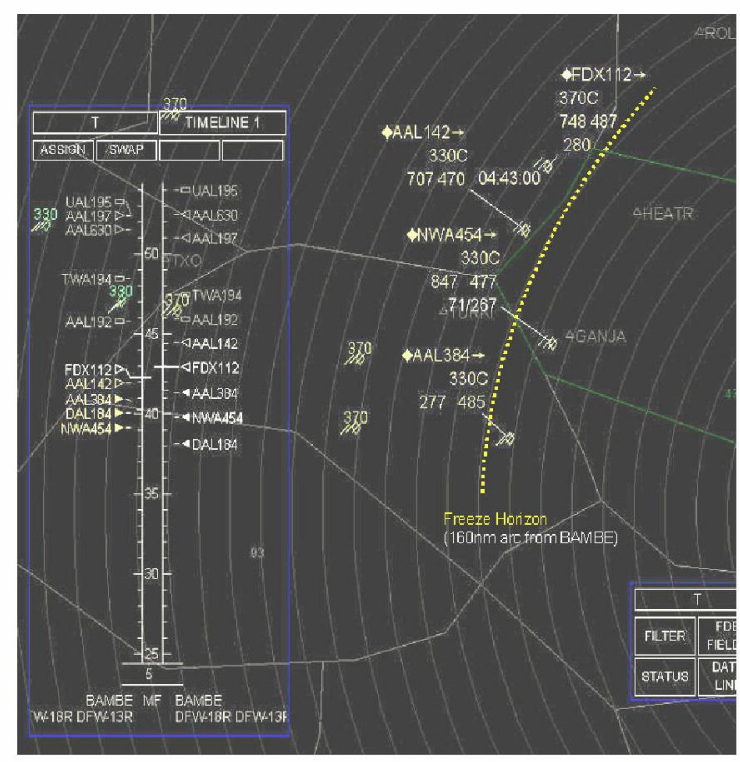

Figure 3. DSR Emulation with Timeline

To maximize the benefits of advanced air and ground-side DSTs, they were integrated with Controller Pilot Data Link Communication (CPDLC) and the Flight Management System (FMS). This integration allows the controllers and the pilots to exchange 4-D trajectory information quickly and with low workload. Much of the capabilities described below, e.g. speed advisories, altitude and route trial plan, etc., were integrated with CPDLC to be able to uplink them to the flight crews as a clearance.

The controller data link interface was modeled after CPDLC Build I used in Miami Center (ZMA). Its features include data block symbology, automated transfer-of-communication (TOC), and a status list. The CPDLC-based TOC was modeled after the process used in ZMA and proceeds as follows. A sector handoff is initiated by the transferring controller. When the handoff is accepted, a frequency change uplink message is automatically sent to the aircraft. The pilots then accept the CPDLC message and change the radio frequency to the appropriate channel. This TOC mode, called "TOC AUTO", was the preferred mode by the controllers in a previous study [7]. 
One of the key capabilities of the implemented DSTs is the integration of trajectory-oriented tools with CPDLC. Trajectory-oriented metering has shown potential benefits in efficiency and workload in handling arrivals. Based on earlier research an initial set of DSTs was recommended [8]. One of those capabilities is an interactive timeline that provides a graphical representation of the meter fix scheduler that is modeled after the Center TRACON Automation Systems (CTAS) Traffic Management Advisor (TMA). The timeline in Figure 3 shows the estimated time of arrivals (ETAs) on the left side and scheduled time of arrivals (STAs) on the right. The STA at the meter fix was automatically assigned once an aircraft was within $160 \mathrm{~nm}$ of BAMBE meter fix during the study, but the frozen STAs can be reassigned afterwards if necessary.

Another trajectory-oriented metering tool is a speed advisory. Speed advisories are computed along an aircraft's current route to deliver it to the meter fix on the STA. In Figure 4, for example, the fourth line in the data block shows a speed advisory of .81 Mach in cruise and 312 knots in descent. The controller may uplink this advisory to the flight deck as a loadable data link clearance. If a speed change alone cannot deliver the plane on its STA, the controller can modify the 4-D flight path using trial plan capabilities to either stretch or shortcut the path or change the aircraft's cruise altitude. During trial planning, the ETA on the timeline is updated dynamically to reflect the ETA changes resulting from the proposed path change. The trial plan capability is accessed by clicking on a trial planning portal (right arrow) on the data block (Figure 4).

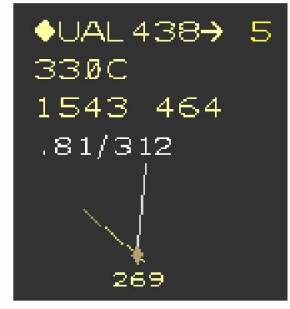

Figure 4. Prototype DSR Data Tag with Trial Planning Portal (Arrow), Speed Advisory, and Predicted Conflict in 5 Minutes

Graphically, the ground-side CD\&R automation indicates a potential LOS in two ways. First, trajectory based conflict-probe (CP) alerts are displayed as minutes to LOS in the first line of the data block. Clicking on the time to LOS highlights the aircraft targets and displays the flight paths and the predicted conflict location (Figure 5). The second alert representing the current day conflict alert (CA) uses an independent state-based logic and triggers data block flashing. Trajectory-based conflict predictions can also be presented in a conflict list. Once a conflict is identified, trial planning can be used to create a new lateral route, a new altitude, or both. The ground-side CD\&R automation is active for the trial planned route/altitude as well as the current route, so the controller can create a conflict-free path before sending it as a clearance via CPDLC.

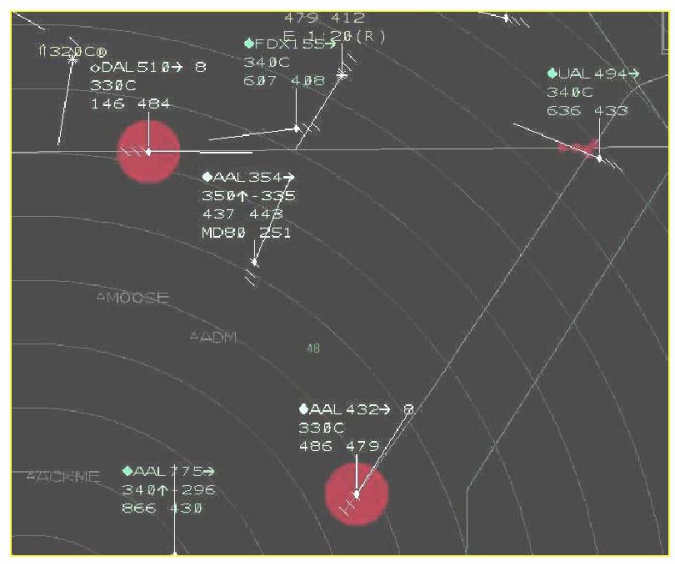

Figure 5. Conflict Probe Display

In this study, AFR aircraft were presented to the controllers as limited datablocks - with callsign, datalink status, and current altitude - in order to limit their impact on workload (Figure 6). Unlike 15 minute look-ahead time for IFR-IFR conflicts, the look-ahead time for AFR-IFR conflicts was only 3 minutes (AFR-AFR conflicts were not shown at all). The short look-ahead time for AFRIFR conflicts was so that these conflicts would have minimal impact on the controller workload unless the impending conflict was not resolved until the last moment. They were shown to controllers only as a safety back-up. 


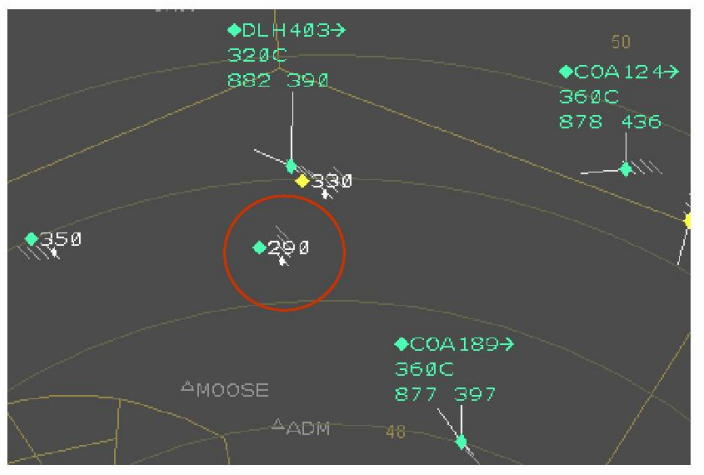

Figure 6. AFR Aircraft Displayed as Limited Datablocks (Highlighted In Red Circle)

Colors were added judiciously on the DSR displays to enhance the ability to monitor the traffic and to allow similar functions to be visually grouped together. In particular, different colors were used for the datablocks of arrivals and overflights/departures which was determined effective in past studies $[7,8]$. In this study, colors were also used to further limit the visual impact of AFR aircraft by picking an appropriate datablock color and intensity for the AFR aircraft to minimize their visual presence on the displays. In the simulation, all aircraft were equipped with CPDLC, FMS, and automatic dependent surveillancebroadcast (ADS-B). The aircraft flown by the commercial pilot participants also had conflict detection $\&$ resolution (CD\&R) as well as advanced required time of arrival (RTA) capabilities.

\section{Ground-Side Results}

The overall air and ground results presented in [5] suggest that the En Route Free Maneuvering concept element has great potential to increase en route and transition airspace capacity, provided that safety concerns raised by controllers can be addressed. Meter fix conformance was equally good for mixed operations and managed operations, suggesting an effective coordination between managed and autonomous flights at the meter fix. The following section will discuss the details of potential capacity gains and safety issues.

\section{Impact of Traffic Volume on Workload}

A primary anticipated benefit of the concept is the ability of en route airspace to accommodate substantial increases in traffic volume through the increase of AFR aircraft. In order to test this hypothesis, the traffic scenarios gradually increased traffic to its maximum during the first twenty minutes of the simulation and maintained this traffic level during the next 30-35 minutes before tapering off for the last 5-10 minutes. Figure 7 illustrates the traffic pattern for Amarillo sector across four conditions. The traffic patterns were similar for the other two high altitude sectors - i.e. Ardmore and Wichita Falls. The graph shows average total aircraft count (i.e. both AFR and IFR) every 5 minutes for the four conditions, as well as the average IFR aircraft count for the mixed equipage conditions $\mathrm{C} 2-\mathrm{C} 4$.

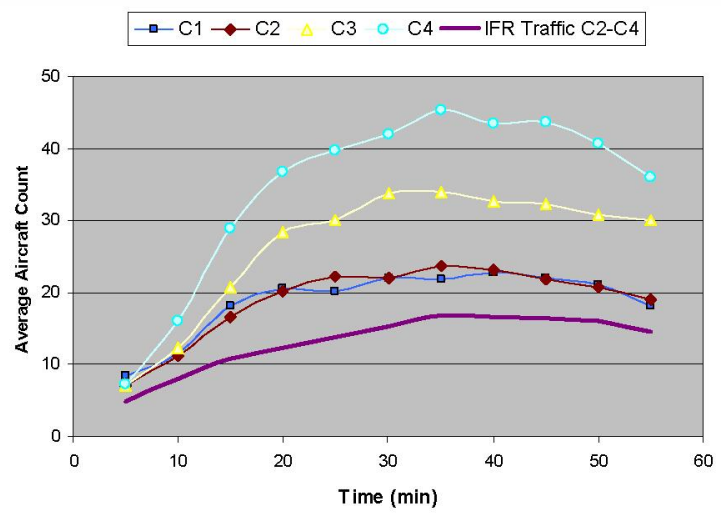

\section{Figure 7. Average Aircraft Count over Time} across Four Experimental Conditions

The targeted traffic levels for Amarillo were 20, 20, 30, and 40 for $\mathrm{C} 1, \mathrm{C} 2, \mathrm{C} 3$, and C4, respectively; for Ardmore, they were 18, 18, 30, and 40; and for Wichita Falls, they were 16, 16, 20, and 24 . The Bowie sector did not have a targeted traffic count as it only handled arrivals to the meter fix, but the arrival rate was set to 84 seconds which allowed 8-10 aircraft to be in sequence. As shown in Figure 8, maximum aircraft counts in each sector exceeded the targeted traffic levels for all conditions. 


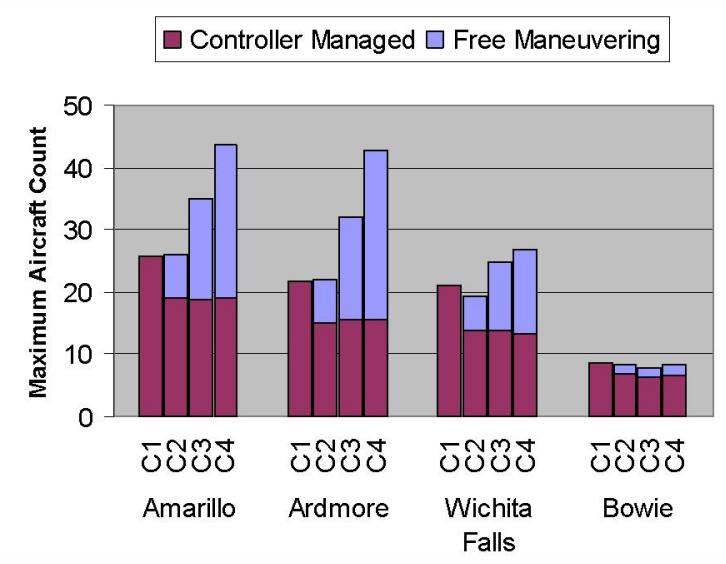

Figure 8. Maximum Aircraft Count in Each Condition per Sector

For the Amarillo sector, maximum count was 26, 26, 35, and 44 for C1-C4; for Ardmore, 22, 22, 32, and 43; for Wichita Falls, 21, 19, 25, and 27; and for Bowie, 9, 9, 8, and 9. For C2-C4 conditions, the IFR portion of the aircraft count was approximately $70 \%$ of the IFR count in the all managed condition $(\mathrm{Cl})$.

Aircraft count in the all managed condition (C1) shows that high altitude controllers were able to work traffic levels well beyond current day MAPs with only one controller managing each position. The relationship between sector count (Figure 8) and workload ( $\mathrm{Cl}$ in Figure 9) provides evidence that these peak counts in all the managed condition resulted in manageable workload. A full integration of air and ground systems via CPDLC by itself showed substantial capacity benefits, even without free maneuvering aircraft. It is likely that without the integrated decision support tools, the manageable peak aircraft count would be substantially lower. By using the advanced ground support tools, it was relatively easy to monitor and maintain separation during the all managed condition $(\mathrm{M}=2.0 ; 1=$ very easy, $5=$ very difficult) and to deliver aircraft on schedule during the all managed condition $(\mathrm{M}=1.5)$.

In mixed operations with high traffic density (i.e. C3 and C4), the controllers managed traffic that far exceeded the current day MAP values, demonstrating the potential en route capacity gains of the concept. Moreover, controller workload appeared to correlate primarily to the number of managed aircraft, whereas the number of autonomous aircraft in the airspace had little impact on controller workload. It is worth emphasizing that these results would not be possible without a tightknit integration of air and ground DSTs via CPDLC since they rely heavily on automation to offload tasks - such as automated handoffs/TOC and reliable conflict predictions - related to AFR aircraft.

Figure 9 shows average workload ratings per sector across the four conditions, which shows a similar pattern as that of IFR portion of the aircraft count in Figure 8. Subjective workload assessments were collected from controllers using the Air Traffic Workload Input Technique (ATWIT) [9]. Controllers were required to rate their workload on a scale of 1 to 7 , at 5-minute intervals throughout each simulation run. The workload ratings showed higher workload for $\mathrm{C} 1$ than those for $\mathrm{C} 2-\mathrm{C} 4$, suggesting that mixed traffic posed no significant workload. Furthermore, the workload was relatively flat for C2-C4 despite a significant increase in AFR traffic, suggesting that AFR aircraft did not create a significant amount of workload.

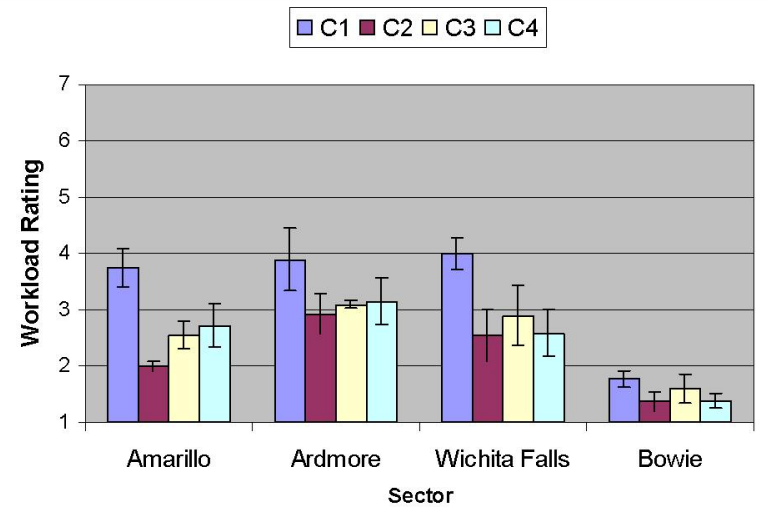

Figure 9. Controller Workload (ATWIT)
Ratings

\section{Traffic Complexity and Safety}

Based on the workload data, one might erroneously conclude that increasing the AFR aircraft count in $\mathrm{C} 3$ and $\mathrm{C} 4$ did not result in increased workload because they added no traffic complexity to the controllers. On the contrary, postrun ratings on traffic complexity reveal that the controllers increasingly rated the traffic to be more complex from $\mathrm{C} 2$ to $\mathrm{C} 4$ (Figure 10). 


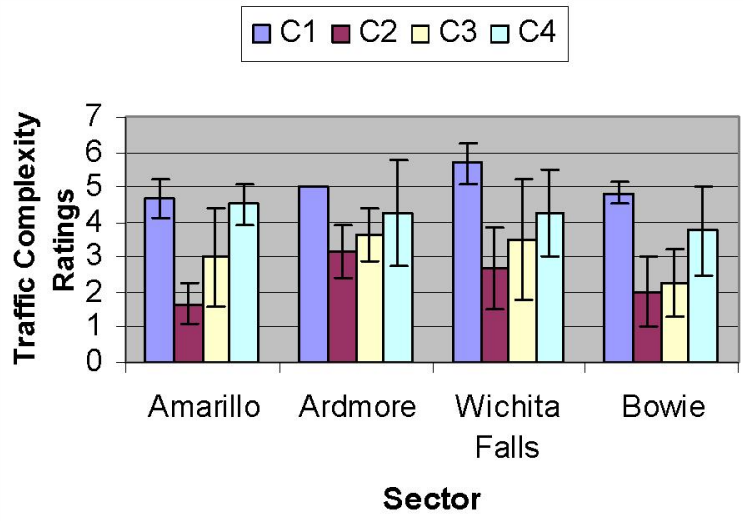

Figure 10. Traffic Complexity Ratings

The data suggest that the controllers were able to dissociate traffic complexity from workload and used workload ratings to indicate only the amount of "activity" that they were engaged in. However, the complexity ratings in $\mathrm{C} 4$ were still lower than those for all managed operations $(\mathrm{Cl})$ despite a gradual increase in traffic complexity ratings from $\mathrm{C} 2-\mathrm{C} 4$, suggesting that the overall traffic complexity was reduced in mixed operations while significantly increasing the aircraft count.

A likely source of increased traffic complexity in $\mathrm{C} 2$ vs. $\mathrm{C} 4$ is the reduced maneuver space for controller-managed IFR aircraft due to the sheer volume of AFR aircraft at the $\mathrm{C} 4$ traffic level. In the high altitude sectors in C4, AFR aircraft often blocked the potential path changes for IFR aircraft, especially during the descent phase of the arrivals. Another source of increased complexity is added display clutter of limited datablocks for AFR aircraft. At the relatively low AFR traffic volume in $\mathrm{C} 2$, the limited AFR datablocks provided peripheral traffic awareness without cluttering the display. However, at the high $\mathrm{C} 4$ traffic level, the sheer volume of AFR aircraft created enough clutter on the display that controllers had some difficulty accessing IFR datablocks.

Another significant source of increased complexity was an increase in AFR-IFR conflicts in higher traffic levels. The ground side tools provided controllers with CP alerts whenever AFR-IFR conflicts were unresolved with less than 3 minutes to LOS. Table 1 tabulates the AFR-IFR conflicts that were alerted to the controllers.
Table 1. AFR-IFR Conflicts with LOS within 3 Minutes $^{1}$

\begin{tabular}{|l|c|c|c|c|}
\hline Conflict Type & C2 & C3 & C4 & Total \\
\hline $\begin{array}{l}\text { Pseudo-piloted } \\
\text { AFR aircraft }\end{array}$ & 13 & 35 & 71 & 119 \\
\hline $\begin{array}{l}\text { Single-piloted } \\
\text { AFR aircraft }\end{array}$ & 15 & 19 & 17 & 51 \\
\hline Total & 28 & 54 & 88 & 170 \\
\hline
\end{tabular}

The increases in unresolved AFR-IFR conflicts were mainly due to pseudo-pilot AFR flights, which had greater difficulty in resolving conflicts as the traffic volume increased. The participant pilots, who flew single-piloted AFR aircraft simulators, seemed to be less affected by the traffic increase. The volume of impending AFR-IFR conflicts that the controllers observed in the high traffic conditions - caused mostly by the limitations in the pseudo-pilot stations or autonomous agent pilots led to their safety concerns.

The increase in the perceived traffic complexity due to increased number of unresolved AFR-IFR conflicts was also reflected in the controller safety ratings. In the post-simulation questionnaire, controllers rated mixed operations less safe than all managed operations $(M=2.25 ; 1$ $=$ much less safe; $5=$ much safer). Controllers also gave pairwise preference comparison ratings between all possible pairs of simulation conditions with respect to overall safety. These ratings were analyzed using the Analytic Hierarchy Process (AHP) statistical technique [10], wherein the preference data for each question is transformed into a percentage and averaged for all controllers to produce numerical ranking scores (Figure 11). As shown in Figure 11, controllers consistently ranked the all managed condition $(\mathrm{C} 1)$ as the safest, followed by L1, L2, and L3-mixed conditions (C2 C4). Follow up discussions suggested that high number of AFR-IFR conflicts contributed heavily to the safety concerns.

\footnotetext{
${ }^{1}$ Due to data logging problems, the analysis includes data from runs 5-16 only.
} 


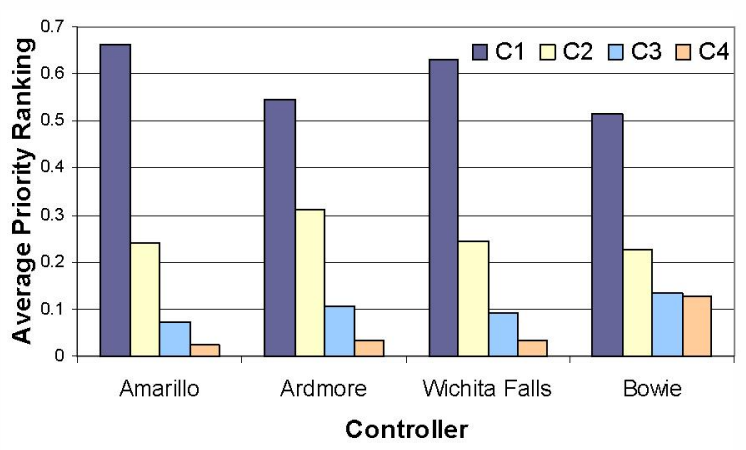

Figure 11. Controller Safety Rankings

The increased safety concerns were not reflected directly by the number of separation errors. Examining the number of losses of separation as a function of safety, there were $3,4,5$, and 7 separation errors for $\mathrm{C} 1-\mathrm{C} 4$, respectively. The data only pertains to aircraft that were controlled by participant pilots or controllers (i.e., AFR flights flown by pseudo-pilots or autonomous agents were excluded). Although the number of violations increased gradually from $\mathrm{C} 1-\mathrm{C} 4$, it is difficult to generalize the results from the number of violations because each violation resulted from a unique circumstance [5]. The number of IFR-IFR violations remained constant with increasing AFR traffic levels, suggesting that the increasing traffic levels of AFR aircraft did not negatively impact controllers' ability to separate IFR aircraft.

\section{Task Load}

The workload reduction under mixed operations confirmed the hypothesis that if the separation responsibility is given to the AFR aircraft pilots, then the controllers can be relieved of the tasks (e.g. handoffs, clearances, etc.) associated to those aircraft without introducing a significant number of new tasks, such as increased monitoring of AFR aircraft or increased number/complexity during conflict-related route modifications.

Some of these findings can be verified by directly measuring the task loads. For example, Figure 12 shows the average number of handoffs that were initiated and accepted during a simulation run. Since AFR aircraft required no manual handoffs by the controllers, the number of handoffs mirrored the managed aircraft count in Figure 8. In addition, pilot check-ins were also not required for
AFR aircraft. Overall, reduction of these route tasks for AFR aircraft seemed to have contributed to the overall reduction in controller workload.

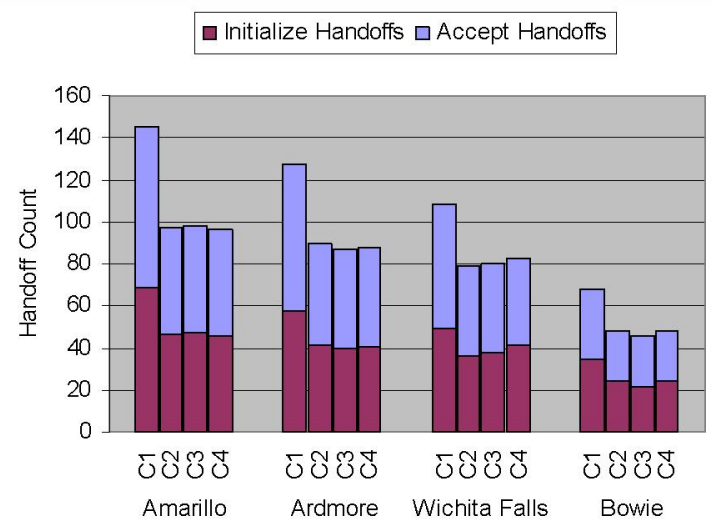

Figure 12. Number of Handoffs

Theoretically, the reduction in handoff-related task loads may be offset by an increased number of route modifications that may be needed to resolve conflicts in high traffic mixed operations. However, Figure 13 shows that the number of route, speed, altitude, and temporary altitude clearances issued by the controllers were actually reduced in all mixed operations. Interestingly, the number of clearances did not increase from $\mathrm{C} 2$ to $\mathrm{C} 4$, even with a significant increase in traffic complexity and reduced maneuver space in $\mathrm{C} 4$ condition. The finding suggests that burdening the AFR aircraft of separation responsibility effectively shifted the task of path changes to the pilots without creating additional workload for the controllers. The air-side data (reported in [11]) showed that the number of conflicts that an AFR aircraft needed to solve nearly doubled from $\mathrm{C} 2$ to $\mathrm{C} 4$, suggesting that the increase in traffic did create more congested airspace with increased potential for conflicts. However, simple altitude or route resolutions were able to resolve most of these conflicts - even at the highest traffic levels - and secondary conflicts were minimal. In sum, overall reduction of route, speed, and altitude clearances seemed to have been another contributing factor to the workload reduction in mixed operations. 


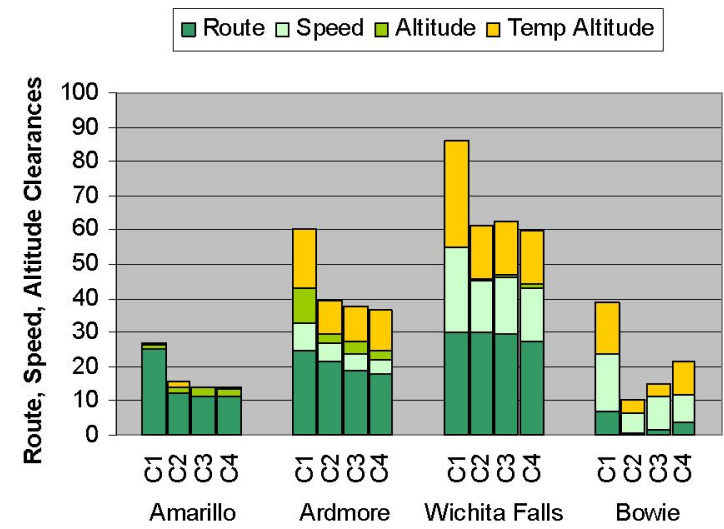

Figure 13. Number of Route, Speed, and Altitude Clearances

Figure 13 also shows that the total number of clearances was highest for Wichita Falls, followed by Ardmore then Amarillo in the three high altitude sectors. The order was reversed for the maximum number of aircraft - i.e. highest for Amarillo, followed by Ardmore then Wichita Falls. This finding suggests that each aircraft in Wichita Falls required more number of clearances, likely due to the sector characteristics (e.g. sector geometry, number of arrival aircraft, etc.) and associated traffic complexity.

Both handoff and clearance data partially explain the reduction in controller workload under mixed operations. Key missing data to complete the overall picture is the task load associated with increased monitoring under mixed operations. For example, one can infer from Table 1 that AFR-IFR conflict monitoring was significantly increased in $\mathrm{C} 4$ condition due to the near-term AFR-IFR conflicts, potentially contributing to increased controller workload. Although accurate ground-side conflict predictions were available in this simulation study, unreliable conflict probe could potentially add to the monitoring task load, especially under mixed operations in which controllers have little or no situation awareness of AFR aircraft. In this study, monitoring task data was not captured but it may be necessary to capture them in future studies with additional data collection mechanisms, such as eye tracking devices.

\section{Meter Fix Conformance}

One challenge for controllers under mixed operations was to manage the STA for all IFR arrivals in the presence of AFR aircraft. The data suggest that the meter fix conformance under mixed operations posed no significant problem, as the percentages of RTA, altitude, and speed conformance for AFR and IFR aircraft exceeded $90 \%$ across all conditions (Figure 14). The results were not statistically significant across the four traffic conditions $(\mathrm{p}>.15)$.

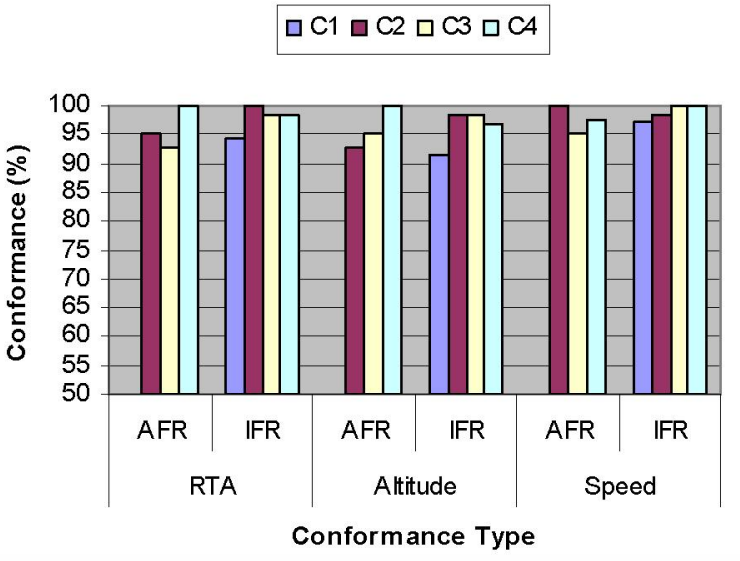

Figure 14. RTA, Altitude, and Speed Conformance at the BAMBE Meter Fix

Overall, controllers did not have significant problems delivering aircraft within \pm 15 seconds of their STA. The number of IFR flights that deviated from the STA was quite small - less than 3\%. AFR pilots also had little difficulty in conforming to the schedule. Arrival conformance varied little regardless of whether the subject-piloted aircraft was AFR or IFR. Similarly, complying with the TRACON crossing restriction of $11,000( \pm 300)$ feet and $250( \pm 10)$ knots was not a particular problem for controllers or AFR pilots. IFR aircraft were equally likely to conform to the crossing restriction in the mixed as well as the all managed condition.

\section{Concept Acceptability - Controller Perspective}

At the end of the simulation, controllers were asked to rate the acceptability of different aspects of the free maneuvering concept. Controllers had a positive impression on metering efficiency. They thought that mixed operations was actually slightly more efficient than all managed operations $(\mathrm{M}=$ $3.5 ; 1=$ much less efficient, $5=$ much more 
efficient) and that it was just as easy to sequence planes in mixed as in managed operations $(\mathrm{M}=3.0$; $1=$ very easy, $5=$ very difficult). They also thought that it was easier to deliver managed aircraft to the meter fix during mixed operations $(M=2)$, likely due to the fact that they had fewer aircraft to manage when some of the arrival aircraft were free maneuvering.

In contrast, they had somewhat negative impressions on situation awareness and safety. They rated mixed operations to be less safe than managed operations ( $M=2.25 ; 1=$ much less safe; $5=$ much safer) and they thought that it was slightly more difficult to detect non-conforming aircraft (M $=3.25$ ). They also thought that it was somewhat more difficult to cope with unplanned events $(M=$ $3.75 ; 1=$ much less difficult, $5=$ much more difficult) and to maintain/monitor separation $(\mathrm{M}=$ $3.25)$

Burdening AFR aircraft to resolve all AFRIFR conflicts was also marginally acceptable $(\mathrm{M}=$ 2.9; 1 = completely unacceptable, $5=$ completely acceptable). When an AFR-IFR conflict was imminent, controllers thought that the procedures and phraseology for resolving the conflict was somewhat unacceptable $(M=2.3)$. However, the phraseology for requesting pilot intent was rated somewhat acceptable $(\mathrm{M}=3.8)$.

The controllers elaborated further when asked about the acceptability of the concept during debrief discussions. In general, controllers' comments highlighted four significant safety issues regarding concept acceptability: automation dependency, situation awareness of AFR aircraft, near-term AFR-IFR conflicts, and overall traffic density.

The first three concerns were specific to AFR aircraft. One of their concerns was that if the conflict detection automation "misses" an AFR-IFR conflict, the conflict may not be independently detected by the controller because they are discouraged from monitoring autonomous aircraft. Although they were not responsible to resolve these conflicts, they felt that they should be able to independently monitor conflicts that may endanger passenger safety. The automation dependency concern has a wider implication when applied to the flight deck automation as well. When the flight deck automation fails to detect conflicts, the consequences are far greater since the pilots do not have the domain expertise to independently monitor the potential conflicts. Therefore the flight crews depend completely on the automation for accurate conflict detection.

Controllers were also concerned with degraded situational awareness of AFR aircraft. In order for AFR flights to not add any workload for the controllers, they need to be nearly invisible to the controllers (e.g., limited depiction on the controller's display, no controller responsibility, little interaction with IFR aircraft). However, if information about AFR traffic is suppressed, the controller is less prepared to provide service for exceptional cases, such as unresolved near-term conflicts and RTA revisions. Less awareness leads to inability for the controllers to deal with emergency situations but more awareness undermines the scalability premise.

Controllers commented extensively on the near term AFR-IFR conflicts. They felt in general that waiting until an IFR-AFR conflict is within 2-3 minutes seems too late to start critical decisions. They also felt that there was the potential for ambiguous information because it was not always clear if the AFR aircraft was taking action to resolve the conflict that it was responsible for. The general feeling of "not knowing" what the AFR aircraft was doing caused additional concern and even when they knew the aircraft intent, they weren't always sure if the intended action was appropriate. One of the key lessons learned from the study was the importance of clear and unambiguous procedures for both pilots and controllers when handling short-term AFR-IFR conflicts. If the resolution responsibility is to be shared between the pilot and controller under these situations, then some level of air and ground system compatibility may be required. Alternatively, if the responsibility is to remain solely with the AFR pilot, then the decision to alert the controller to these conflicts should be re-visited.

Finally, an interesting point raised by the controllers was that the current day rules and procedures have excess buffers built in to absorb errors by the controllers and/or by the system. It might not be good idea to strip away all of the safety buffers by dramatically increasing the traffic density. They were concerned that increased traffic density reduced options for maneuvering IFR 
aircraft out of critical situations. One controller commented that "...resolution was always more difficult in high mixed environment because AFR aircraft are in the way of IFR aircraft." In general, they were not sure how one determines what capacity increases can be achieved without compromising safety. One controller commented that "our reality is people fly planes, people work planes, and people get on planes", so safety should be valued higher than efficiency because people's lives are at stake.

\section{Decision Support Tools and Display}

The results from this simulation demonstrated potential capacity benefits with the free maneuvering concept. A critical requirement for achieving this benefit, however, was a full integration of air and ground systems via CPDLC, which significantly reduced controller workload by offloading tasks to the automation (e.g. TOC via CPDLC), maintaining an efficient traffic flow (e.g. timeline), and keeping aircraft on 4D trajectories (e.g. trial plans, route, altitude, and speed uplinks) for better conflict prediction and intent inference.

Therefore, development of usable tools to support these functionalities was critical to the concept implementation. Controller ratings of tool usability and usefulness suggest that the tools which supported the critical tasks indeed performed well during the experiment. Average usefulness ratings ranged from 3.5-5.0 ( $1=$ Not useful, $5=$ Very useful) and average usability ratings ranged from $3.0-5.0(1=$ Very difficult to use, $5=$ Very easy to use). Table 2 shows the top six items on the usability/usefulness ratings, which consist of ground-side tools that support 4-D trajectory operations (e.g. trial planning tool, trial plan conflicts), efficient traffic management (e.g. speed advisories, timeline), and clearances via CPDLC.
Table 2. Controller Rating of Usability and Usefulness of Displays and Tools

\begin{tabular}{|l|c|c|}
\hline Tool Feature & Useful & Usable \\
\hline Trial-planning tool & 5 & 5 \\
\hline Speed advisories & 5 & 4 \\
\hline $\begin{array}{l}\text { Graphical display of trial } \\
\text { plan conflicts }\end{array}$ & 4.8 & 4.5 \\
\hline CPDLC interface for TOC & 4.8 & 4.5 \\
\hline $\begin{array}{l}\text { CPDLC interface for } \\
\text { clearances and requests }\end{array}$ & 4.8 & 4.3 \\
\hline Arrival timelines & 4.6 & 4.3 \\
\hline
\end{tabular}

The trial-planning tool received a considerably higher usability rating than in the 2002 simulation (2004 usability rating $=5.0,2002$ usability rating $=$ 3.0) [8]. The redesigned highly responsive trial planning tool integrated with the R-side display provided immediate conflict feedback. Full CPDLC integration for easy uplink of trial plans allowed the controllers to work the traffic without issuing many vectoring instructions. In this study, controllers thought that trial plan route and altitude amendments were much more effective than vectoring and altitude changes in current day operations ( 4.75 and 4.25 , respectively; $1=$ much less effective, $5=$ much more effective). One controller commented that tools allowed controllers to plan and make more efficient decisions.

In addition, controllers rated that data link clearances greatly reduced their workload $(\mathrm{M}=$ 4.67; 1 = greatly increased, $5=$ greatly reduced). CPDLC's reduction of frequency congestion was a very useful workload reduction tool. The lowest combined usability and usefulness rating was for the graphical display of AFR-IFR conflicts ( $M=$ $3.0, M=3.5$, respectively). Controllers commented that frequent AFR-IFR conflict alerts lead to display clutter, partly because the alerting method involved displaying the AFR aircraft's expanded data blocks.

The questionnaire also asked about the preferred display location of delay absorption, conflict, and data link status information on either the datablock and/or on lists/timeline. In general, all controllers thought that critical information on the 
datablock was essential since lists in general added to display clutter and were often ignored when busy. Unlike lists, controllers were able to effectively use the timeline and thought that it was useful. Although too much information on the display could have been a problem, none of the controllers voiced any issues with the IFR datablocks in the simulation.

\section{Conclusion}

The joint Ames/Langley simulation study of the DAG-TM En Route Free Maneuvering concept element demonstrated potential en route capacity benefits. When the majority of the aircraft were free maneuvering, the total aircraft count far exceeded the current day MAPs in the high altitude sectors. In these high traffic situations, controller workload remained manageable and was actually lower than those of managed operations with more IFR but fewer total aircraft. The data suggest that workload is correlated primarily with the managed portion of the traffic, at least up to twice the maximum current day traffic, validating one of the key assumptions that AFR aircraft has minimal workload impact on the controllers.

Despite reporting manageable workload with high traffic levels of mixed traffic, controllers reported increasing traffic complexity imposed by the additional AFR aircraft. At the highest traffic level, AFR aircraft limited the potential maneuver space for IFR aircraft and caused display clutter even though they were shown as limited datablocks that took little display space. Increased AFR traffic also increased the number of AFR-IFR conflicts mostly due to limitations of multi-aircraft stations and/or autonomous agent pilots. These conflicts were main contributors to safety concerns and increased complexity ratings by the controllers.

Mixed operations would not have been feasible without a well integrated air/ground system that connects Flight Management Systems, airborne decision support tools, traffic flow management tools for scheduling and trajectory planning, ground-based decision support tools, integrated CDPLC/DSTs, and broadcast of up-to-date state and short-term intent information. In this paper, we focus on the impact of the ground-based DSTs on the success of the overall concept. The ground DSTs have been significantly re-designed from our past studies to improve the responsiveness and accuracy of the tools. The design of individual display components has also been significantly improved. The integrated air/ground system and the corresponding decision support tools described here are a key component to excite maximal benefits in many of the future concepts that are discussed today. Therefore, the tools, procedures, results, and lessons learned from this study and simulation architecture should provide a solid foundation to test different concepts in the future.

\section{Acknowledgment}

Distributed Air-Ground Traffic Management (DAG-TM) research is funded by the Airspace Systems program as part of the Advanced Air Transportation Technologies Project (AATT). The simulation described in this paper owes its success to many dedicated individuals at AATT project office, NASA Ames Flight Deck Display Research Laboratory, Airspace Operations Laboratory, Crew Vehicle Systems Research Facility, and NASA Langley Air Traffic Operations Laboratory. We are also grateful for the assistance of members of BoozAllan Hamilton, and Titan Systems, who contributed long hours to this project. This work could not have taken place without the active support of the Air Line Pilots Association, the National Air Traffic Controllers Association, and the Air Traffic Services Office of the Federal Aviation Administration. The authors appreciate their interest in and support of our research.

\section{References}

[1] NASA, 1999, Concept Definition for Distributed Air/Ground Traffic Management (DAG$T M)$, http://www asc.nasa.gov/aatt/dagconop.pdf

[2] Erzberger, H., 2004, Transforming the NAS: The Next Generation Air Traffic Control System, $24^{\text {th }}$ International Congress of the Aeronautical Sciences, Yokohama, Japan, August 2005.

[3] RTCA, 1995, Final Report of RTCA Task Force 3:Free Flight Implementation, Washington, DC.

[4] Eurocontrol, 2003, CoSpace Delegation of Spacing Tasks from Air Traffic Control to Flight Deck, http://www.eurocontrol.fr/projects/freer 
[5] Barhydt, R. and Kopardekar, P., 2005, Joint NASA Ames/Langley Experimental Evaluation of Integrated Air/Ground Operations for En Route Maneuvering, The Sixth International Air Traffic Management R\&D Seminar ATM-2005, Baltimore, MD, July 2005 .

[6] Lee, P. U., Mercer, J., Smith, N., \& Palmer, E., 2005, A Non-Linear Relationship between Controller Workload, Task Load, and Traffic Density: The Straw that Broke the Camel's Back, Proceedings of the 13th International Symposium on Aviation Psychology, Oklahoma City, OK, April 2005 .

[7] Lee, P. U., D'Arcy, J. F., Mafera, P., Smith, N., Battiste, V., Johnson, W., Mercer, J., Palmer, E. A., Prevot, T., 2004, Trajectory Negotiation via Data Link: Evaluation of Human-in-the-loop Simulation, International Conference on Human-Computer Interaction in Aeronautics, Toulouse, France, September 2004.

[8] Prevot T., Lee, P., Callantine, T., Smith, N. and Palmer, E., 2003, Trajectory-Oriented Time-Based
Arrival Operations: Results and Recommendations, ATM2003, FAA/Eurocontrol R\&D Seminar, Budapest, Hungary.

[9] Stein, E, 1985, Air Traffic Controller Workload: An Examination of Workload Probe.,

DOT/FAA/CT-TN84/24. DOT/FAA Technical Center. Atlantic City, NJ.

[10] Saaty, T., 1980, The Analytic Hierarchy Process, New York, McGraw Hill.

[11] Johnson, W., Battiste, V., Granada, S., Johnson, N., Dao, A. Q., Wong, D., and Tang, A., 2005, A Simulation Evaluation of a HumanCentered Approach to Flight Deck Procedures and Automation for En Route Free Maneuvering, International Symposium on Aviation Psychology, Oklahoma City, OK.

$24^{\text {th }}$ Digital Avionics Systems Conference October 30, 2005 